\title{
Decoding the matrix: multiomics reveals host-microbe biomarker for inflammatory bowel disease
}

\author{
Monica Viladomiu and Randy S. Longman \\ Jill Roberts Institute for Research in IBD, Division of Gastroenterology and Hepatology, Department of Medicine, Weill Cornell Medicine, New York, New York, USA.
}

\begin{abstract}
Inflammatory bowel disease (IBD) is a chronic inflammatory disease of the intestine associated with genetic susceptibility and alterations in the intestinal microbiome. Multiomics data developed and analyzed over the last several decades have yielded an unprecedented amount of genetic and microbial data. But how do we pinpoint mechanistic insight into the hostmicrobe relationship that will ultimately enable better care for patients with IBD? In this issue of the $J C I$, Grasberger et al. undertook a major decoding effort to decipher this multiomic data matrix. The authors analyzed anonymized data from more than $\mathbf{2 8 0 0}$ individuals to discover a link between heterozygous carriers of deleterious DUOX2 variants and high levels of plasma IL-17C. These findings provide an example of how harnessing big data can drive mechanistic discovery to define disease biomarkers that have the potential to improve clinical care in IBD.
\end{abstract}

\section{Finding the host-microbiome link}

For many of us in the IBD community patients, scientists, and physicians - finding the underlying link between host and microbe driving intestinal inflammation is the holy grail of our research. Understanding this relationship has the power to not only reveal the underlying etiology of disease, but also to stratify patients with genetic susceptibility, identify new disease biomarkers, and select precise medical therapies to guide safer and more effective treatment. In this issue of the JCI, Grasberger et al. provide a critical window into the matrix of multiomics data (1).

Grasberger and colleagues used phenome-wide association study (PheWAS) and corresponding plasma metabolite data from 2872 participants to computationally identify (via sequence kernel association tests) low-frequency proteinaltering DUOX2/DUOXA2 gene variants associated with high plasma IL-17C levels. DUOX2 encodes a subunit of an inducible, epithelial-specific NADPH oxidase that regulates the release of hydrogen peroxide as part of the innate defense response to normal gut microbiota and potential pathogens. Using a heterologous system to functionally test the relevance of these variants, the authors identified highimpact variants that diminished NADPH oxidase assembly at the membrane surface, reduced hydrogen peroxide production critical to its innate immune function, and correlated with increased circulating IL-17C (1).

This portal linking DUOX2/DUOXA2 variants with IL-17C also provides mechanistic insight into how host physiology can shape the mucosa-associated microbe compartment (Figure 1). Previous work from this group and others (2-4) has established the role for apical release of hydrogen peroxide in shaping mucosa-associ-

\section{Related Article: https://doi.org/10.1172/JCl141676}

Conflict of interest: RSL serves as a consultant for Bristol Myers Squibb and Pfizer and has received research funding from Boehringer Ingelheim.

Copyright: () 2021, American Society for Clinical Investigation.

Reference information: J Clin Invest. 2021;131(9):e148902. https://doi.org/10.1172/JCl148902.

ated bacteria. Grasberger and colleagues reasoned that defective hydrogen peroxide production in heterozygous carriers of deleterious DUOX2 variants may increase epithelial access by gram-negative bacteria. To test this idea, the authors used mice with a deficiency in Duoxa. Consistent with their hypothesis, mucosa-associated (but not luminal) Proteobacteria species were substantially expanded. This enrichment was specific to gram-negative bacteria, as a relative loss of gram-positive segmented filamentous bacteria (SFB) was also observed. The authors further pinpoint the function of DUOX2 to the mucus layer. Treatment with carboxymethylcellulose, an emulsifier that degrades the mucus barrier, abrogated the impact of DUOX2. This finding functionally reflects the mucosal thinning seen in IBD-associated intestinal inflammation and helps explain the uniform finding reported by many groups regarding the bloom of Enterobacteriace$a e$ and the particularly adherent-invasive E. coli seen in Crohn's disease (1).

\section{IL-17C as a marker of intestinal dysbiosis and inflammation in Crohn's disease}

In addition to mucosal microbe regulation by host genetics, Grasberger and colleagues found additional specificity of this link to Crohn's disease. First, the correlation between epithelial-specific Duoxa deficiency and higher IL-17C expression was seen only in the terminal ileum, the site most commonly affected in Crohn's disease, and not the colon. Second, the authors found a differential ability of commensal microbes to induce IL-17C. While ileal-adherent SFB robustly induced the Duoxa pathway and triggered a conventional Th17 response, SFB (and other gram-positive bacteria) failed to induce IL-17C. Using epithelial cell organoids, the authors showed the specificity for gram-negative bacteria in triggering IL-17C independently of Duoxa. Thus, epithelial DUOX2 and hydrogen peroxide pro- 
Healthy individual

Homeostasis

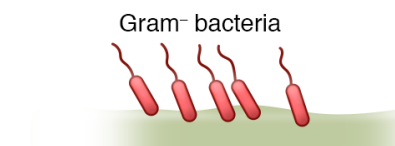

Mucus

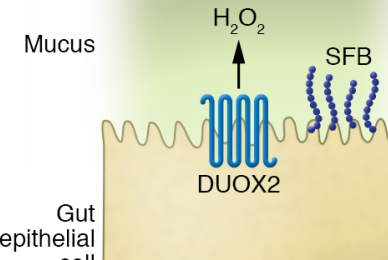

epithelial
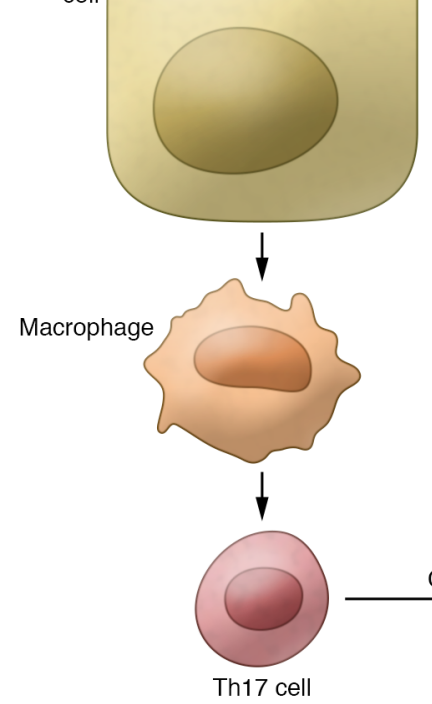

Th17 cell
Individual with IBD

Microbial dysbiosis

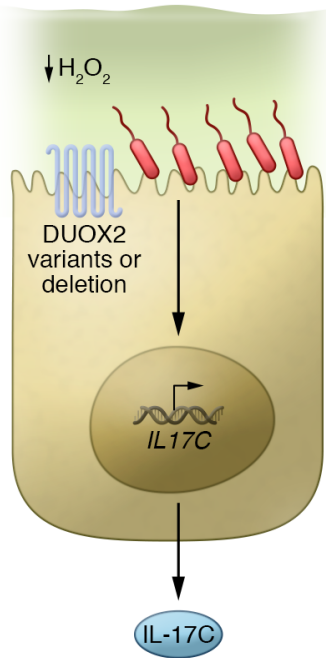

Plasma biomarker with potential to guide therapy i i 1 CCL20

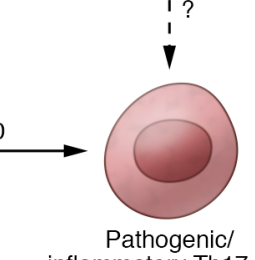

inflammatory Th17 cell

Figure 1. Deleterious DUOX2 variants regulate barrier immunity and reveal IL-17C as a plasma biomarker of microbial dysbiosis in IBD. In healthy individuals, production of hydrogen peroxide by DUOX2 promotes innate barrier immunity. In IBD patients with deleterious DUOX2 variants, a reduction in hydrogen peroxide allows for gram-negative penetration of the mucus barrier and induction of IL-17C secreted through the basolateral surface. IL-17C is increased in IBD patients with gram-negative dysbiosis and has the potential to serve as a biomarker of disease.

duction shape the repertoire of mucosaassociated microbiota while IL-17C reflects the specificity of gram-negative expansion in the absence of hydrogen peroxide secretion (1).

Another clinically important aspect of these findings is that the basolateral release of IL-17C renders it a potential biomarker of microbial dysbiosis and an early marker of intestinal inflammation in Crohn's disease. Grasberger and colleagues offered several critical lines of evidence to support this point. First, the level of IL-17C expression correlated with mucosal expansion of gram-negative Proteobacteria species in mice lacking DUOX2 activity. Second, aberrant circulating IL-17C was associated with disease in the treatment-naive Crohn's disease RISK cohort. Analysis of the mucosa-associated microbiota in the RISK cohort further strengthened the positive correlation between gram-negative microbiota and plasma IL-17C. Although the authors noted that IL-17C $\mathrm{C}^{\text {hi }}$ status was not unique to carriers of deleterious DUOX 2 variants, this phenotype reflects a degenerate pathway (including DUOX2 deficiency) that enables gram-negative activation at the ileal surface (1). This type of surrogate biomarker could be critical in providing noninvasive monitoring of intestinal inflammation desperately needed for the clinical management of Crohn's disease.

To further test the clinical relevance of these high-impact $D U O X 2$ variants, Grasberger et al. used three large IBD cohorts stratified according to ancestry-specific allele frequency. Pooled meta-analysis revealed a significant odds ratio (OR) of 1.54 , with the strongest OR driven by the Ashkenazi Jewish cohort. Although the cumulative population attributable risk (PAR) for protein-altering $D U O X 2$ variants was only $0.68 \%$ (compared with $1.6 \%$ for the most significant NOD2 variants), these variants could meaningfully contribute to what's known as the "missing heritability" in IBD. Moreover, these studies implicate a functional role for low frequency $D U O X 2$ loss-of-function variants and the subsequent microbial dysbiosis in the underlying pathogenesis of IBD.

\section{Guiding IBD therapy}

Given these findings, key questions remain on how to integrate data from Grasberger et al. (1) to optimize treatment and personalize medication selection. First, could plasma IL-17C levels identify a subset of patients with IBD who would benefit from antimicrobial treatment or priority for fecal microbial transplantation (FMT)? The current use of either antibiotics or FMT remains investigational in the treatment of IBD and stratification of subjects with microbe-driven disease may enhance treatment efficacy. Second, could deleterious variants of DUOX2 or plasma IL-17C levels serve as markers of early or aggressive disease? Predicting disease behavior remains a major clinical challenge with a huge potential impact on selecting either a top down approach with early use of biologic therapy versus a step up approach as disease becomes more severe. Many recent studies have established a role for gene variants, DNA methylation, and serum proteins in the behavior of Crohn's disease $(5,6)$. Patient stratification based on DUOX2 variants may help refine these algorithms. Finally, can DUOX2 status or plasma IL-17C levels help predict patient response to biologic or small molecule therapies? It is interesting to see the correlation of plasma IL-17C with CCL2O, which suggests the potential to feedback and enhance Th17- and IL-23-mediated disease. Could this correlation reflect an increased sensitivity to IL-23 blockade? Or will anti-TNF- $\alpha$ treatment block the synergy with IL-17C? Clinical data evaluating medication response will help guide the impact of the Grasberger et al. (1) findings 
on our current management algorithms for IBD. Decoding the matrix of IBD multiomics may not occur instantly, but this stepby-step progress provides critical windows into this wealth of data that will help guide more precise, effective, and safe therapy for the treatment of IBD.

\section{Acknowledgments}

RSL is supported by NIH grants DKR01114252 and DKR01-120985. MV is supported by a Research Fellowship Award from the Crohn's and Colitis Foundation.
Address correspondence to: Randy S. Longman, 413 E. 69th Street, New York, New York 10021, USA. Phone: 646.962.6289; Email:ral2006@med.cornell.edu.

1. Grasberger H, et al. DUOX2 variants associate with preclinical disturbances in microbiota-immune homeostasis and increased inflammatory bowel disease risk. JClin Invest. 2021;131(9):e141676.

2. Collins KD, et al. Chemotaxis allows bacteria to overcome host-generated reactive oxygen species that constrain gland colonization. Infect Immun. 2018;86(5):e00878-17.

3. Grasberger $\mathrm{H}$, et al. Dual oxidases control release of hydrogen peroxide by the gastric epithelium to prevent Helicobacter felis infection and inflammation in mice. Gastroenterology. 2013;145(5):1045-1054.

4. Pircalabioru G, et al. Defensive mutualism rescues NADPH oxidase inactivation in gut infection. Cell Host Microbe. 2016;19(5):651-663.

5. Somineni HK, et al. Blood-derived DNA methylation signatures of Crohn's disease and severity of intestinal inflammation. Gastroenterology. 2019;156(8):2254-2265.

6. Wu J, et al. Serum protein biomarkers of fibrosis aid in risk stratification of future stricturing complications in pediatric crohn's disease. Am J Gastroenterol. 2019;114(5):777-785. 